OPEN ACCESS

Edited by:

Raquel Montenegro,

Federal University of Ceara, Brazil

Reviewed by:

Rosalinda Sorrentino, University of Salerno, Italy

Wan-Wan Lin,

National Taiwan University, Taiwan

*Correspondence:

Jun Lyu

lyujun2020@jnu.edu.cn

Specialty section:

This article was submitted to Pharmacology of Anti-Cancer Drugs,

a section of the journal

Frontiers in Pharmacology

Received: 02 November 2021 Accepted: 10 January 2022

Published: 27 January 2022

Citation:

Li T, Liu H, Dong C and Lyu J (2022) Prognostic Implications of PyroptosisRelated Gene Signatures in Lung

Squamous Cell Carcinoma. Front. Pharmacol. 13:806995. doi: 10.3389/fphar.2022.806995

\section{Prognostic Implications of Pyroptosis-Related Gene Signatures in Lung Squamous Cell Carcinoma}

\author{
Tingting $L i^{1}$, Huanqing $L_{i u^{2}}$, Chunsheng Dong ${ }^{3}$ and Jun $L y u^{2 *}$ \\ ${ }^{1}$ Department of Pharmacy, Xi'an Chest Hospital, Xi'an, China, ${ }^{2}$ Department of Clinical Research, The First Affiliated Hospital of \\ Jinan University, Guangzhou, China, ${ }^{3}$ School of Computer Science, Shaanxi Normal University, Xi'an, China
}

Background: Lung squamous cell carcinoma (LUSC) has been a highly malignant tumor with very poor prognosis. It is confirmed that pyroptosis refers to the deaths of cells in a programmed and inflammatory manner. Nevertheless, the correlation between expression of genes related with pyroptosis and their prognosis remains uncertain in LUSC.

Methods: Utilization of The Cancer Genome Atlas (TCGA) cohort has been done for evaluating the prognostics of pyroptosis-related genes for survival and constructing a signature with multiple genes. The least absolute shrinkage and selection operator (LASSO) Cox regression was performed for establishing such pyroptosis-related gene signature.

Results: Eventually, identification of 28 genes in relation to pyroptosis was made in LUSC and healthy lung tissues. Upon the basis of these differentially-expressed genes (DEGs), the patients of LUSC can be divided into two subtypes. Nine gene signatures were established using LASSO. The surviving rate for low-risk group was apparently greater in contrast with the high-risk group $(p<.001)$. According to our finding, risk score worked as an independent predictive factor of OS among LUSC sufferers in combination with clinical characteristics. In line with Gene Ontology (GO) and Kyoto Encyclopedia of Genes and Genomes (KEGG) analyses, the enrichment of immunity-related genes and decreasing immunity status among the high-risk group.

Conclusion: Genes in relation with pyroptosis played an essential role in tumor immunity, which is capable of predicting the prognosis for LUSCs.

Keywords: pyroptosis, lung squamous cell carcinoma, prognosis, TCGA, immune microenvironment

Abbreviations: CASP, cysteine-aspartic proteases; DEG, differentially expressed genes; GO, Gene Ontolog; GSDM, Gasdermin; KEGG, Kyoto Encyclopedia of Genes and Genomes; LASSO, The least absolute shrinkage and selection operator; LUSC, Lung squamous cell carcinoma; NK, nature killer; OS, overall survival; PCA, principal component analysis; ROC, receiver operating characteristic; ssGSEA, single-sample gene set enrichment analysis; TCGA, The Cancer Genome Atlas. 


\section{INTRODUCTION}

Pulmonary carcinoma, the most serious malignant tumor, has been emphasized as a predominant reason for carcinoma death occurring in both developed and developing nations in the world (Mattiuzzi and Lippi, 2020). Current statistics have shown that 2.21 million patients were diagnosed with lung carcinoma in 2020; moreover, approximately 1.8 million patients had died from lung carcinoma, ranking as the highest mortality rate of all cancers (Ferlay et al., 2020). Despite radiation and targeted therapies, the survival from lung cancer has not clearly improved, the overall 5 years surviving rate remains under $20 \%$ (Siegel et al., 2018). Such phenomenon has seriously impacted human health, and lung cancer has attracted the public attention to health. As a common histologic subtype of lung carcinoma, LUSC is often not promptly diagnosed clinically and has inherent resistance to radiation and chemotherapy due to its early symptoms is not typical. The lack of appropriate targeted drugs leads to the poorer prognosis of LUSC than that of lung adenocarcinoma (Hirsch et al., 2017). Up to now, prognosis prediction of LUSC still mainly relies on pathological diagnosis and tumor stage system. However, traditional approaches are incapable of accurately evaluating prognoses for LUSC sufferers. Establishing a novel and reliable prognostic model is important to improve the quality, prognosis and OS of patients with LUSC.

Research on programmed cell death has recently attracted considerable attention. Pyroptosis is a programmed cell death associated with the release of an inflammatory molecule in response to stimuli, such as a pathogenic microorganism or chemotherapeutic agent, during which the cell membrane is perforated by the Gasdermin (GSDM) family protein (Galluzzi et al., 2018). Pyroptosis's function in tumor has caused wide concern. Pyroptosis, as a new cellular death type, has exerted both positive and negative effects concerning pathogenesis and treatment of tumors. For one thing, different stimulating factors induce cell pyroptosis to form an inflammatory microenvironment during tumorigenesis, making normal cells transform into tumor cells (Karki and Kanneganti, 2019). For another, pyroptosis has inhibitory function in preventing tumors from occurring and developing (Nagarajan et al., 2019). Explorations have shown the relation amid the potent proinflammatory effect of pyroptosis and the regulatory effect of tumor immunity microenvironment. Gasdermin D (GSDMD) expression deficiency was in the company of a sharp reduction in quantity and activity of CD8 + T lymphocytes (Xi et al., 2019). Pizato et al. (Pizato et al., 2018) have reported that pyroptosis was closely associated with breast cancer cell death and further contributes to the improvement of breast cancer treatment. Some researchers have revealed the positive correlation between p53 and pyroptosis in NSCLC tissues, and p53induced pyroptosis could significantly inhibit tumor growth and improve symptoms and survival of NSCLC to a certain extent (Zhang et al., 2019).

Pyroptosis has a significant function in the development of tumor and antitumor processes, such as liver cancer, breast cancer and stomach cancer (Saeki et al., 2009; Wang et al., 2013; Hergueta-Redondo et al., 2014). Ye et al. (Ye et al., 2021) has demonstrated that pyroptosis-related genes had a significant function in predicting prognosis of ovarian cancer, but few studies have focused on its specific functions in LUSC. Therefore, we studied the expression levels of pyroptosis-associated genes in healthy lung and LUSC tissues in a systematical manner, for exploring the prognosis of these genes as well as investigating the correlation of pyroptosis with the tumor immune microenvironment.

\section{METHODS}

\subsection{Pyroptosis-Related Gene Datasets and Patient Samples}

Extraction of 33 pyroptosis-related genes out of prior reviews was conducted (Man and Kanneganti, 2015; Wang and Yin, 2017; Karki and Kanneganti, 2019; Xia et al., 2019; Ye et al., 2021). Download of the RNA-seq and patients with LUSC was done out of The Cancer Genome Atlas (TCGA) (https:// portal.gdc.cancer.gov/repository). 507 RNA-seq (42 normal and 465 tumor) samples were obtained. With utilization of the "limma" package, DEGs possessing a $p$ value $<.05$ were identified.

\subsection{Establishment and Verification of the Pyroptosis-Associated Gene Prognosis Model}

In order to evaluate the prognosis of pyroptosis-associated genes, utilization of Cox regression analyses was further done for evaluating the correlation between surviving state and each gene in the TCGA cohort.

For preventing negligence, the truncated $p$ value had been adjusted to 0.05 and nine surviving-related genes were screened out for additional analyses. Then, LASSO Cox regression model (R-package "glmnet") was used for narrowing the scope of alternative genes and establishing a prognosis model. Retention of nine genes together with their coefficients was conducted; while, the penalty parameter $\lambda$ was determined according to the minimal criterion. Centralized standardization was carried out for TCGA expression data, and utilization of the scale function in $\mathrm{R}$ was done for calculating the risk score. The risk score formula is shown here: risk score $=$ (coefficient mRNA1 $\times$ expression of mRNA1 $)+($ coefficient mRNA2 $\times$ expression of mRNA2 $)+\cdots$ + (coefficient mRNAn $\times$ expression mRNAn). In line with the median risk score, division of LUSC sufferers was performed as low-risk subgroup and high-risk subgroup; and the OS time between two subgroups was analyzed and compared by Kaplan-Meier analysis. PCA analyses were conducted upon the basis of the characteristics of nine genes. Plotting of a timedependent receiver operating characteristic (ROC) curve should be accomplished for predicting the precision of the prognosis indicators for LUSC sufferers. 


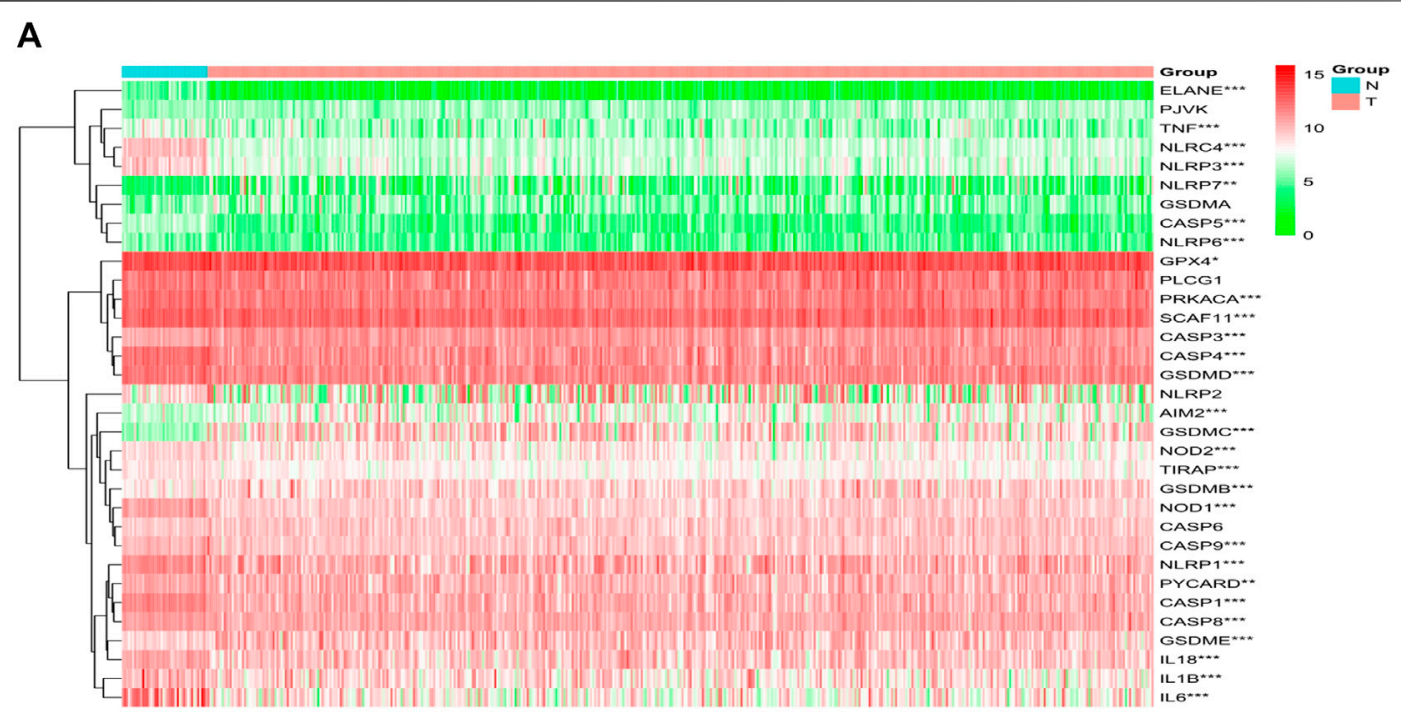

B

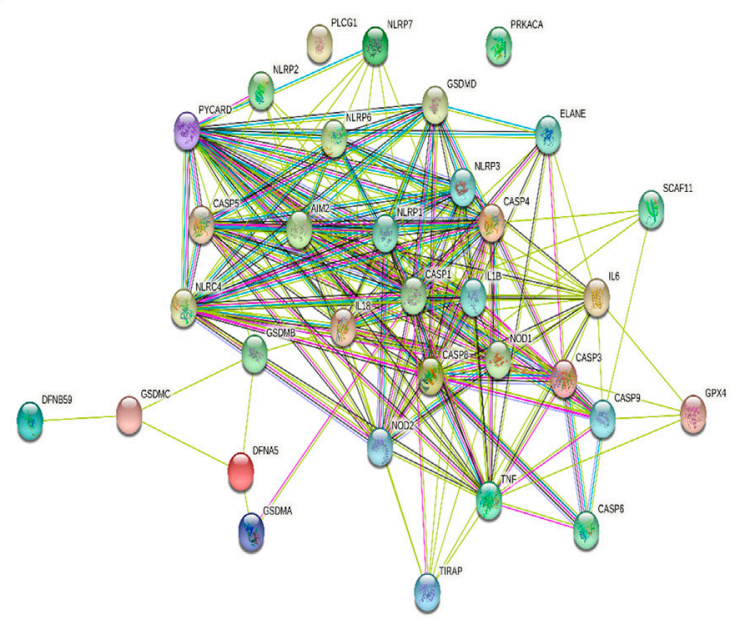

C

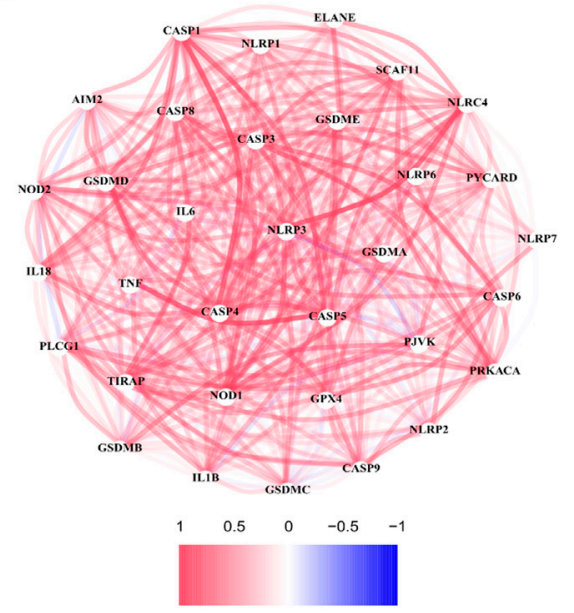

FIGURE 1 | Expression and interaction of DEGs. (A) Heatmap of DEGs amid normal tissue (N, bright blue) and tumor tissue (T, red) (green: low expression level; Red: high expression levels). ${ }^{\star \star} p<.01 ;{ }^{\star \star \star} p<.001$. (B) PPI network showed the interaction of DEGs (interaction score $=0.4$ ). (C) DEGs correlation network (red line: positively correlated; blue line: negatively correlated. The depth of the color gives a reflection on the strength of the correlation).

Functional enrichment analyses of the DEGs amid the lowrisk and high-risk groups.

Division of LUSC sufferers in the TCGA cohort was performed as two subgroups upon the basis of median risk score. According to the certain standard $\left|\log _{2} \mathrm{FC}\right| \geq 1$ and FDR $<0.05$, the DEGs between the two groups were screened. On this basis, utilization of "clusterProfiler" package was conducted for analyzing Gene Ontology Analysis (GO) and Kyoto Encyclopedia of Genes and Genomes (KEGG). "gsva" package was employed for performing single-sample gene set enrichment analyses (ssGSEA), calculating the score of infiltrated immunity cells and evaluating the activity of immunity-associated pathways.

\subsection{Statistics Analyses}

Single-factor ANOVA had been performed for comparing gene expression levels amid healthy tissues and LUSC tissues, and with Pearson $\chi^{2}$ test, a comparison of taxonomical variables was carried out. For comparing the patients' OS rates between the subgroups, Kaplan Meier methodology and two side log-rank test were utilized. Univariate and multivariate Cox regression models had been applied for assessing the independent prognostics for the risk model. With utilization of Mann Whitney test, a comparison of the immunity cellular infiltration and immunity pathway activation was performed amid the two groups. Statistics analysis was performed using $\mathrm{R}$ software (V4.0.2).

\section{Resluts}

\subsubsection{Authentication of DEGs Between the Healthy and Tumor Tissues}

From the TCGA data of 42 healthy and 465 tumor tissues, a comparison of 33 pyroptosis-associated gene expression levels was made and 28 DEGs were identified. Among these DEGs, 22 genes were downregulated (i.e., ELANE, IL6, NLRC4, 


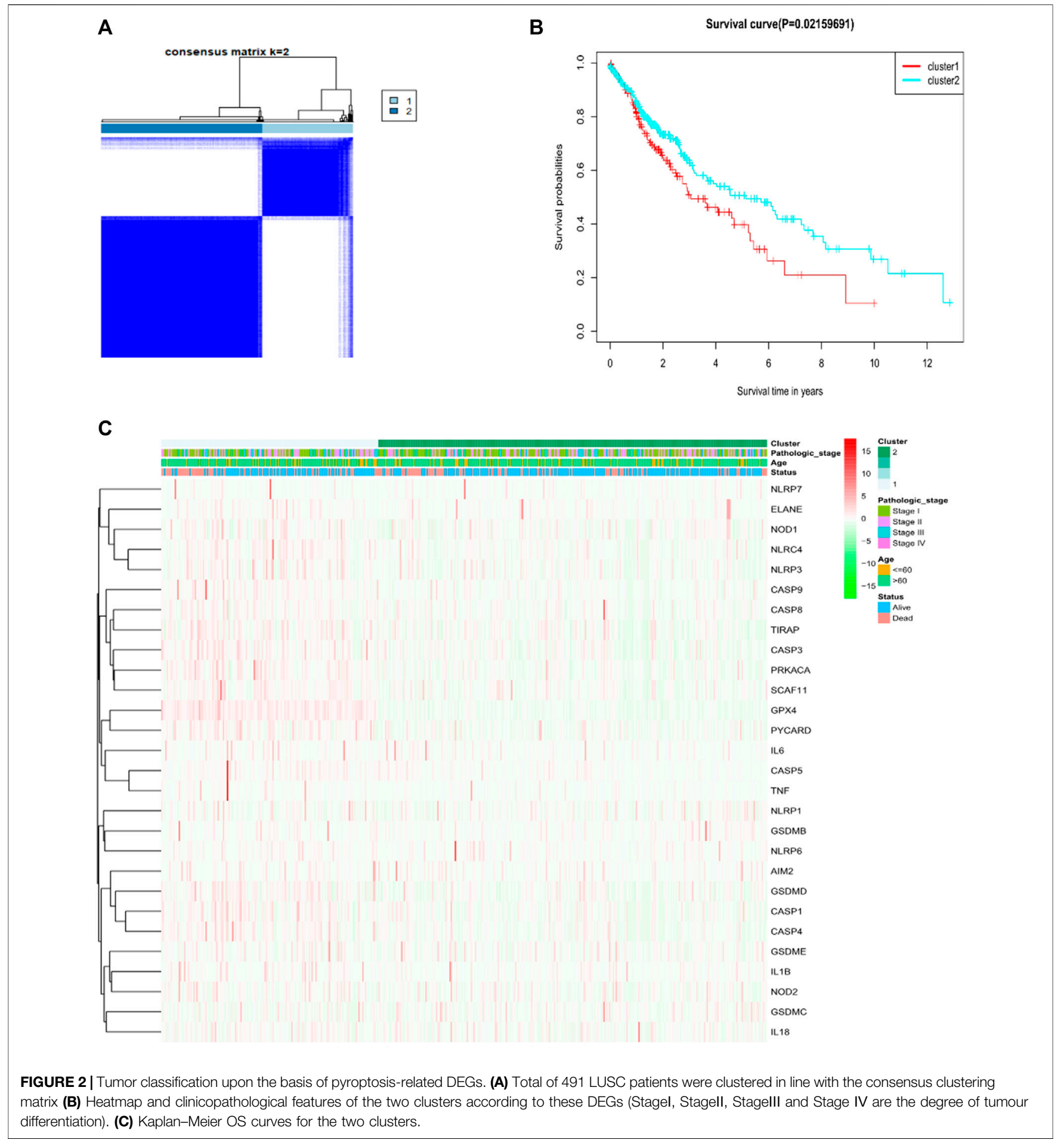

NLRP3, CASP5, IL1B, NOD1, CASP1, CASP4, TNF, NLRP1, TIRAP, NLRP6, IL18, GSDMD, PRKACA, NOD2, CASP9, CASP8, SCAF11, PYCARD, and GPX4), while upregulation happened to six other genes in the tumor group (i.e., CASP3, GSDMB, GSDME, AIM2, NLRP7, and GSDMC). The RNA levels of the genes are shown in Figure 1A. For further exploring the interacted status of pyroptosis-associated genes, a Protein Interaction (PPI) analysis was performed, as indicated in Figure 1B. Through setting of the minimal required interactive score as 0.4 (medium confidence) for PPI analysis, CASP1, PYCARD, NLRC4, NLRP1, CASP5, NLRP3, CASP8 and AIM2 were identified as hub genes according to our determination. Apart from CASP1 gene, all the others were DEGs amid healthy tissue and tumor tissue. The 


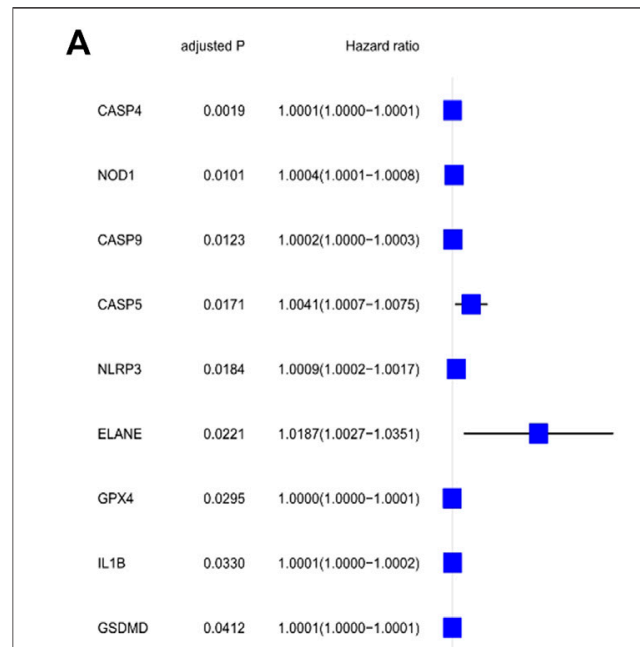

B

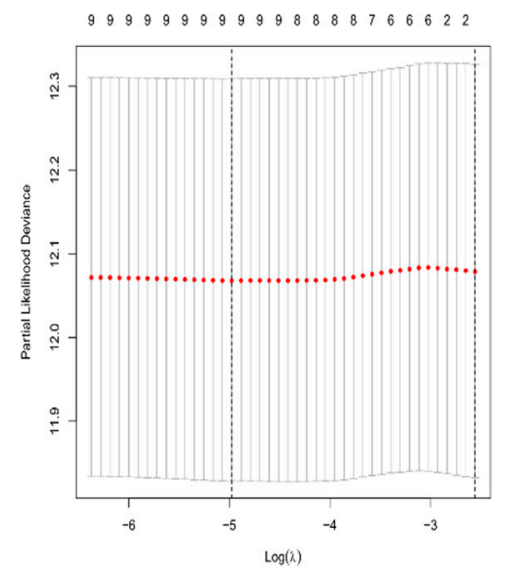

C

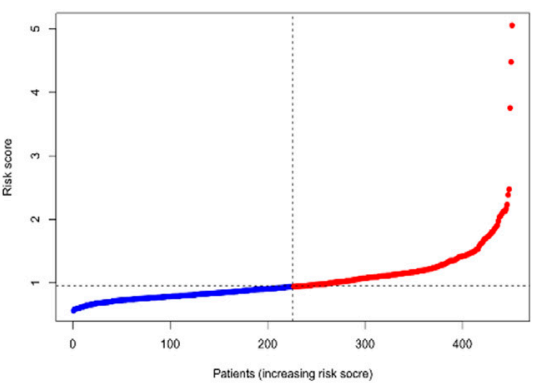

D

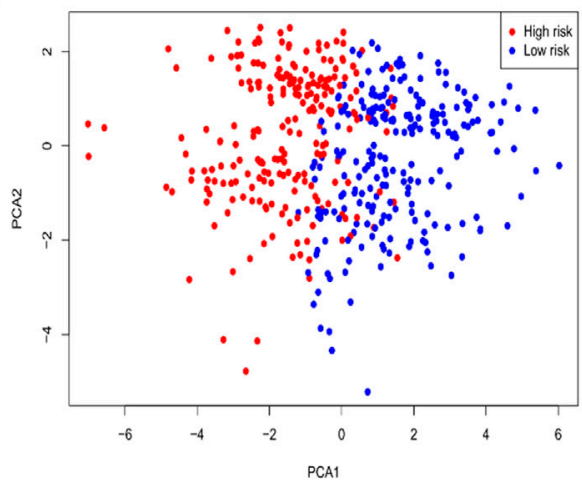

$\mathbf{F}$

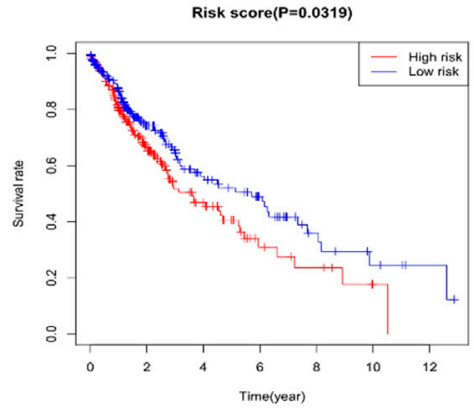

E

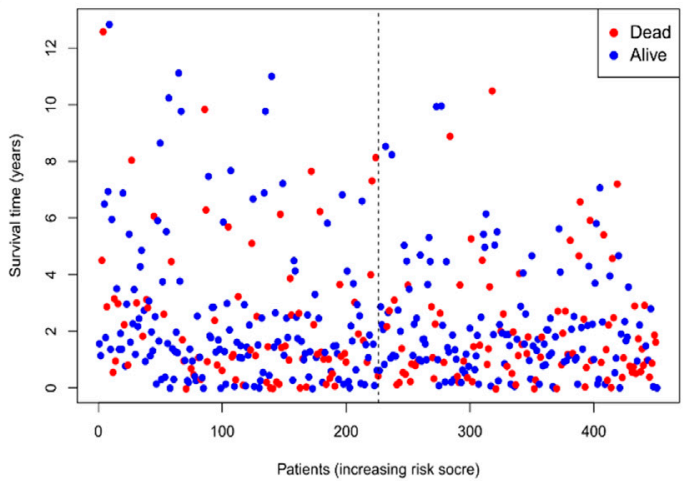

G

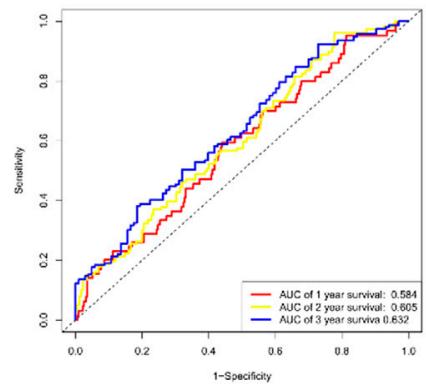

FIGURE 3 Establishment of risk signature in the TCGA cohort. (A) Univariate Cox regression analysis was carried out for the OS of all the pyroptosis-related genes, nine of which were $p$ <.05 (B) LASSO performed regression analysis on nine OS-associated genes. (C) Patients distribution upon the basis of risk score (D) PCA plot for LUSC upon the basis of risk score. (E) Surviving state of all patients (low-risk population: dashed line left; High-risk group: right of dotted line). (F) Kaplan-Meier curves for the OS of patients in the high- and low-risk group (G) ROC curves manifested the prediction effect of the risk score.

correlated network comprising each gene in relation with pyroptosis is shown in Figure 1C.

\subsection{Tumor Classification Upon the Basis of the DEGs}

For investigating the relationship amid the expression of the 28 pyroptosis-associated DEGs and LUSC subtypes, consistent cluster analyses on total 491 LUAD sufferers in the TCGA cohort were done. Through elevating the clustering variable $(\mathrm{k})$ amid 2 and 10, the greatest correlation within group and low correlation amid groups were revealed when $\mathrm{k}=2$, suggesting the practicability of dividing the patients as two clusters according to 28 pyroptosis-related DEGs (Figure 2A). Protein expression profiles and clinical characteristics included heat maps of the degree of tumor differentiation (stages I-IV), age ( $\leq 60$ years or 
$>60$ years), and surviving state (survival or death). However, we found little difference in the clinical characteristics amid two clusters (Figure 2B). Overall survival time (OS) had been found amid the two groups $(p=0.022$, Figure 2C).

\subsection{Construction of a Prognostics Gene Model}

For investigating the effect of the pyroptosis-associated DEGs on LUSC prognosis, Cox univariate analysis (Figure 3A) had been done. Through utilization of LASSO Cox regression model, we selected of genes with the greatest prediction as prognosis indexes. At the time that the median of the sum of squares of residuals was the smallest, $\lambda$ was selected. A ninegene signature was done in line with the optimal $\lambda$ value (Figure 3B). CASP4, NOD1, CASP9, CASP5, NLRP3, ELANE, $G P X 4, I L 1 B$, and GSDMD were identified as prognostic factors for LUSC. Thus, the formula for our model was as follows: Risk Score $=(2.154 \mathrm{e}-05 \times$ expression CASP 4$)+(1.740 \mathrm{e}-04 \times$ expression NOD1) $+(9.836 \mathrm{e}-05 \times$ expression CASP9 $)+$ $(1.433 \mathrm{e}-03 \times$ expression CASP5 $)+(9.719 \mathrm{e}-05 \times$ expression $N L R P 3)+(1.006 \mathrm{e}-02 \times$ expression $E L A N E)+(1.652 \mathrm{e}-05 \times$ expression GPX4) $+(7.975 \mathrm{e}-05 \times$ expression $I L 1 B)+$ (8.574e-06 $\times$ expression GSDMD). In addition, calculation of the risk score for all patients was done in the study cohort. Then, division of this cohort was performed as high-risk group and low-risk group, employing the median risk score as the cut-off value (Figure 3C). As shown in Figure 3D, the sufferers possessing various risks could be divided into the two groups by principal component analysis (PCA). More deaths and less surviving times were seen in the high-risk group versus the low-risk group (Figure 3E). Significant difference was in OS time amid the low-risk group and high-risk group ( $p<.05$, Figure 3F). The ROC curves were also applied for investigating whether the expression patterns of the pyroptosis-associated DEGs could predict the prognosis of LUSC. The AUC values for the 1 year, 2 years, and 3 years survival were $0.584,0.605$, and 0.632 , respectively (Figure 3G).

\subsection{Independent Prognostics for the Risk Model}

Through univariate and multivariate Cox analyses, the possibility of using risk factors as an independent prognosis factor was known. Based on the characteristics of the nine pyroptosis-related genes, risk score $(\mathrm{HR}=1.3777,95 \% \mathrm{CI}: 1.0149-1.8703, p<.05)$ was shown as an independent prognostics for LUSC (Figure 4A). Multivariate analyses revealed the possibility of using risk score as a prognostic factor after adjusting for other confounders (HR $=1.3862$, 95\% CI: 1.0213-1.8813, $p<.05$; Figure 4B). Additionally, a heat map of the clinical characteristics of the TCGA cohort were generated, as shown in Figure 4C, finding that the distribution in the age and surviving status of patients was different amid the low-risk subgroup and high-risk subgroup $(p<.05)$.

\subsection{Functional Analyses Upon the Basis of the Risk Model}

We used limma R package for further exploring the classification of risk model subgroup differences between the gene function and pathways. In the TCGA cohort, altogether 1,637 DEGs were found in the low-risk group and in-risk group, among which upregulation was seen in 1,628 genes and downregulation was seen in nine genes. (Supplementary Table S1). GO enrichment analysis and KEGG pathway analysis had been conducted among DEGs. Results showed main association of DEGs with leukocyte migration, regulation of immune effector process, and cytokinecytokine receptor interaction (Figures 5A,B).

\subsection{Combination of the Immune Activity in the Subgroups}

Upon the basis of functionality analysis, ssGSEA was further performed. The enrichment fractions of 16 immune cells and the activities of 13 immune-associated pathways were compared aimed the low-risk and high-risk populations in the TCGA cohort. According to Figure 6A, compared with the low-risk subgroup, besides the nature killer $(\mathrm{NK})$ cells, the infiltrative level of immunocytes in the high-risk subgroup was generally lower. Additionally, all 13 immune-associated pathways had less activity in high-risk group versus low-risk group (Figure 6B).

\section{DISCUSSION}

Globally, about $85 \%$ of pulmonary carcinoma patients have been confirmed with NSCLC, most of which are hard to cure because of complicated progressive diseases. Chemotherapy based on medication may provide a modest extension of survival for those sufferers. However, the efficacy of current treatments appears to be stagnating without apparent improvement in response rates or median surviving status (Schiller et al., 2002; Scagliotti et al., 2008; Drilon et al., 2012). Numerous studies have been conducted on LUSC, but this cancer is associated with poorer clinical outcomes compared with lung adenocarcinoma (Hirsch et al., 2017).

Pyroptosis, a manner of cellular deaths in a programmed and inflammatory form, causes cellular swelling, plasma membrane dissolution, chromatin rupture and release of the proinflammatory substances in cells. Activated pyroptosis causes the release of inflammatory mediators, contributing to the development and progression of carcinoma ( $\mathrm{Hu}$ et al., 2010). Many experiments have been performed to confirm the role of pyroptosis in carcinogenesis, such as hepatic cellular cancer, breast cancer, and gastric cancer (Saeki et al., 2009; Wang et al., 2013; Hergueta-Redondo et al., 2014). The manner by which the genes associated with pyroptosis interact with each other in LUSC and whether they are related to patient survival are uncertain.

\subsection{Nine Pyroptosis-Related Genes Predicted the Prognosis of LUSC}

We identified a trait with nine pyroptosis-related genes (i.e., GSDMD, CASP4, CASP5, CASP9, ELANE, NOD1, NLRP3, 


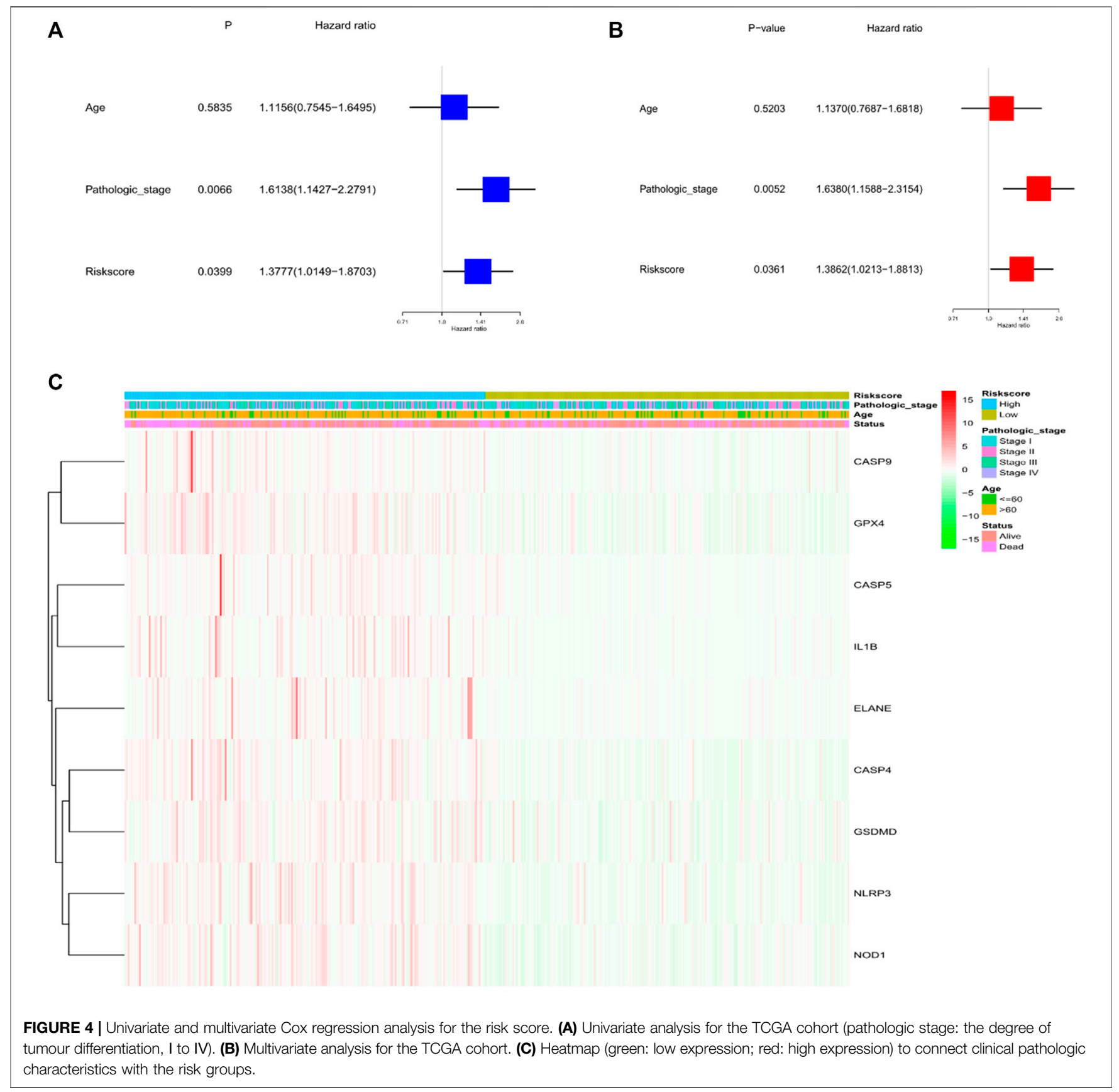

GPX4, and $I L 1 B$ ) and found that these genes had the function of predicting OS in LUSC patients.

GSDMD belongs to a structurally and evolutionarily conserved superfamily of GSDM proteins (Saeki et al., 2009) and has been identified as the key executioner of pyroptosis (Shi et al., 2017). The homologous member of the GSDM family (GSDMA-C, DFNA5) was found in reports to play a potential role in various tumors (Gao et al., 2018). Low expression was found in GSDMD in GC cell lines and models, additionally explorations have shown that down-regulation of GSDMD is capable of regulating the expression of cellular cycle-related proteins and promote tumor cell growth (Qiu et al., 2017). Gao et al. observed the overexpression of GSDMD in LUAD (Gao et al., 2018), suggesting that GSDMD might have a special function in various cancers. Our exploration found GSDMD appeared as an oncogene, because it was upregulated in the tumor tissue. However, it helped to prolong patients' surviving status because of its enrichment in the low-risk group. In consideration of the limitation in information available from LUSC and the conflicts in outcomes for various tumors, the outcomes on GSDMD offer several opinions on deeper research.

Caspases (cysteine-aspartic proteases) are proteolytic enzymes that are used primarily to control cell death and inflammation. 

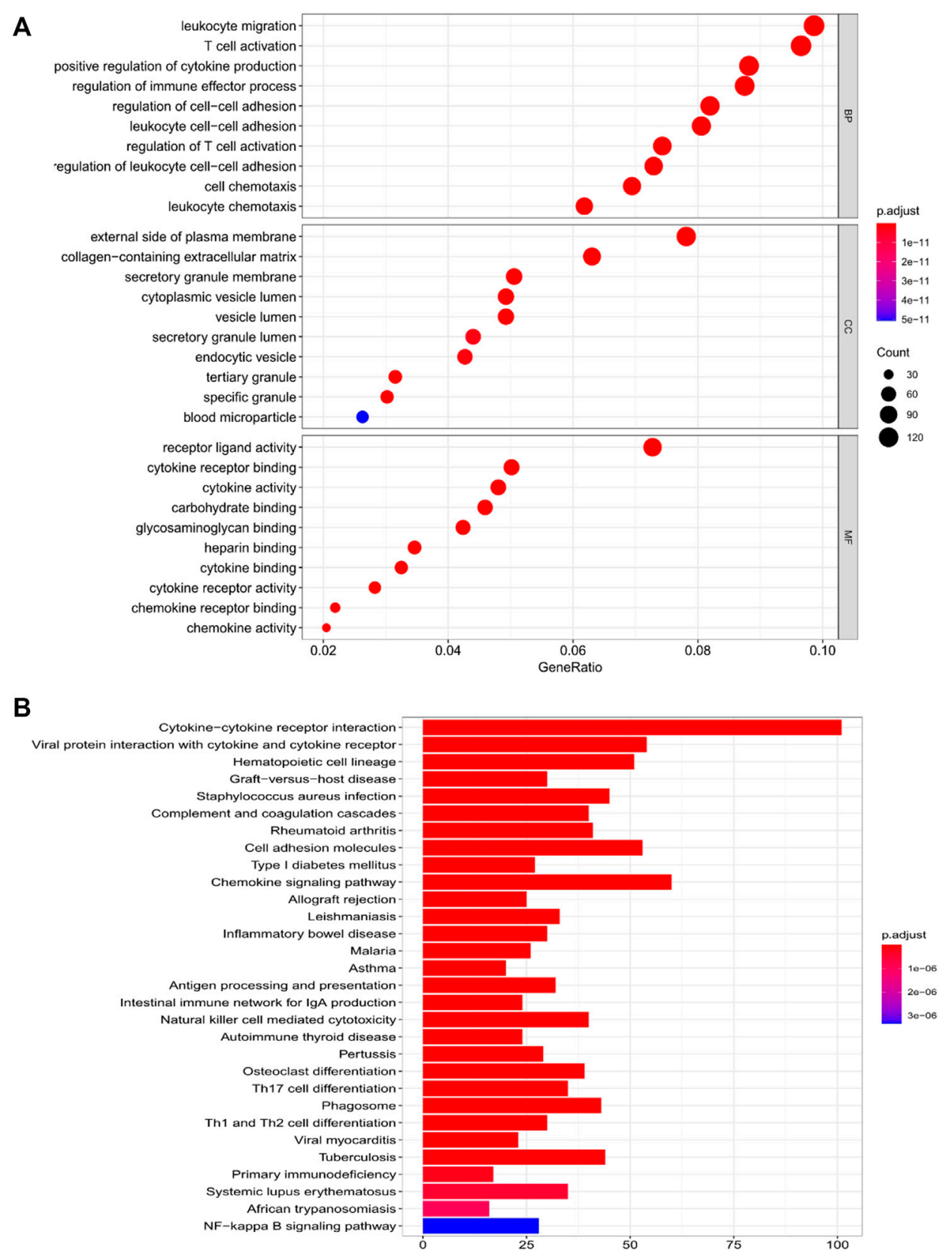

FIGURE 5 | Functional analysis of genes that differed amid the two risk groups in the TCGA cohort. (A) Bubble diagram of GO enrichment (the larger the bubble is, the greater number of genes are enriched; the deeper the red depth is, the obviously greater the difference is). (B) KEGG pathway Barplot (the longer the bar is, the greater number of genes are enriched, and the deeper the red is, the obviously greater the difference is).

Several caspases have been found in mammals, such as human CASP1, CASP4, CASP5, and CASP12, which were identified to be essential mediators for innate immunity responses (Martinon et al., 2004). CASP4 and CASP5 are human homologous genes of mouse CASP11 (Shi et al., 2015; Zhao et al., 2018). Many conclusions about immune function of CASP4 and CASP5 have been based on the studies of CASP11 in mice. CASP 4 has been shown to be directly involved in LPS sensing and served as a key factor in pyroptosis and CASP 1 -mediated IL- $1 \beta$ production in LPS-transfected human monocytes (Baker et al., 2015). CASP4 gene silencing was found to protect THP-1 and U937 monocytes from cytoplasmic LPS-induced pyroptosis (Shi et al., 2014). CASP5 has a synergistic effect with CASP4. Interestingly, CASP4, CASP5, and CASP11 can process GSDMD independently of NLRP3 and its adaptor protein ASC ( $\mathrm{He}$ et al., 2015). By contrast, CASP9 has not been directly associated with inflammatory responses (Galluzzi et al., 2016). However, Abe et al. (Abe and Morrell, 2016) reported that CASP9 

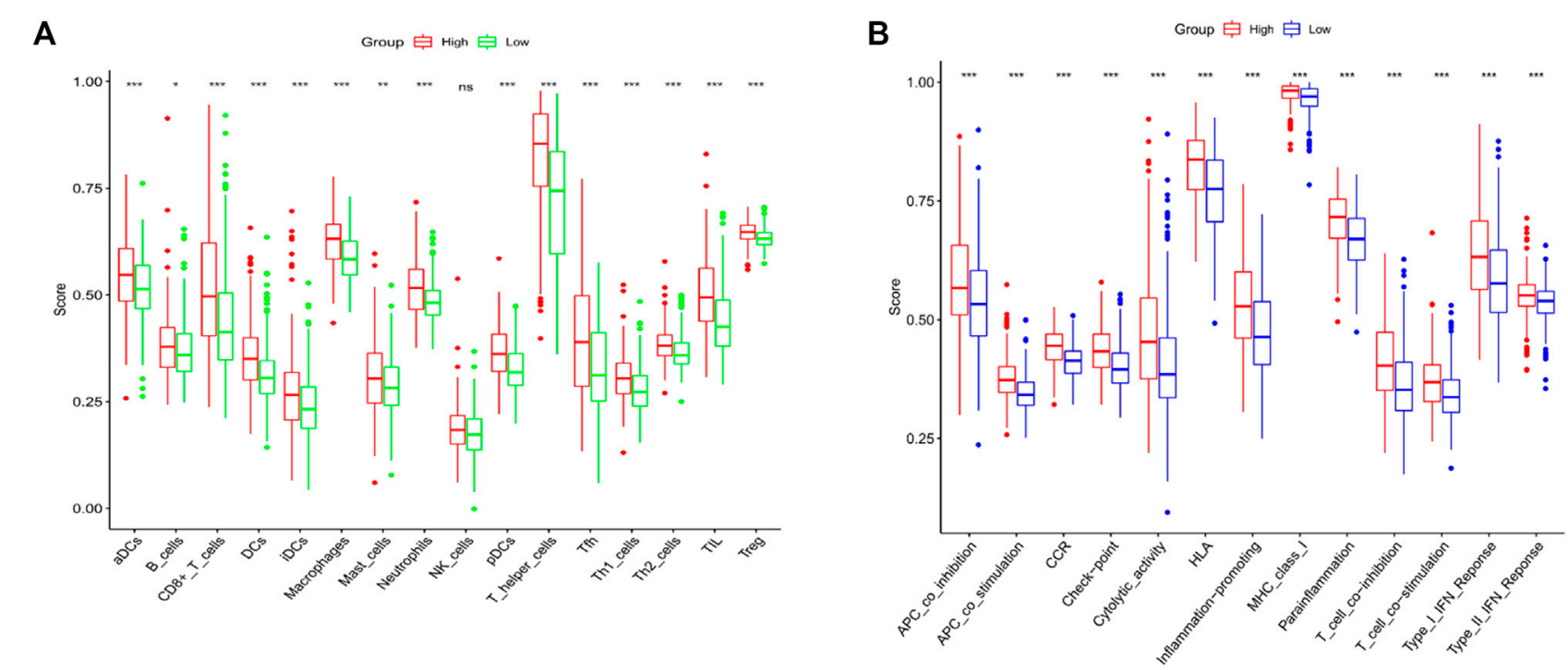

FIGURE 6 | Contrast of SSGSEA scores of immune cells with immune pathways. (A) Contrast was made of the enrichment scores for 16 immunity cells in the low (green box) group and the high risk (red box) group in the TCGA cohort. (B) Contrast of enrichment scores of 13 immune-associated pathways in low (blue box) and high risk (red box) groups in the TCGA cohort.

inhibitors significantly inhibited PI+/Annexin V cell pyroptosis, and its mechanism needs further exploration. These three screened caspase genes, CASP4, CASP5 and CASP9, also have a significant function in antitumor. Qiao et al. (Qiao et al., 2019) demonstrated that a-NETA has induction effect upon pyroptosis of epithelial ovarian carcinoma cells through the GSDMD/ caspase-4 pathway. A previous study has identified tumorassociated CASP4 as a new diagnosis, prediction and prognosis biomarker for NSCLC patients (Terlizzi et al., 2018), nevertheless, the relation of the CASP4-mediated pyroptosis with LUSC development is still unknown. At the same time, according to our findings, high caspase- 4 expression had association with inferior surviving prognosis, which might become the outcomes of its negatively regulated pyroptosis. As an apoptotic initiator protease, CASP9 is involved in tumor process, and Kim et al. (Kim et al., 2015) considered CASP9 as a therapeutic target for treating cancer. According to our analytical results, CASP4, CASP5, and CASP9 were upregulated in the tumor tissues, and their high expression indicated poor survival rate. These results suggested that they played a role as tumor-promoting genes in this study.

ELANE, a protease encapsulated in the main particle of the neutrophil precursor, could activate pro-inflammatory cytokines, comprising TNF- $\alpha, I L-1 \beta$, and $I L-18$ (Fu et al., 2020). Kambara et al. (Kambara et al., 2018) demonstrated that GSDMD was under the cleavage and activation of ELANE for inducing pyroptosis of neutrophils. The more highly expressed ELANE was seen in the high-risk group than the low-risk group, while the neutrophil infiltration scores were apparently lower versus the low-risk group. These results may be due to the activation of the neutrophil focal death by ELANE.

NOD1 belongs to the NOD-like receptor family (Correa et al., 2012). NOD1 mutations are closely related to inflammatory diseases in humans, and a close relationship exists between inflammation and tumor (Layunta et al., 2018). Previous studies have confirmed that single nucleotide polymorphism of NOD1 affects the occurring and advancement of various tumors, comprising lung carcinoma, stomach carcinoma, colorectal carcinoma, pancreatic carcinoma, head and neck squamous cellular carcinoma, etc (Cotterchio et al., 2015; Ozbayer et al., 2015; Suarez et al., 2015).

NLRP3 is one of the most characteristic proteins in the inflammatory bodies of the NLR protein family, which has been proved to have relation with the occurring and advancement of carcinoma. NLRP3 inflammosomes enhance the differentiation of gastric cancer cells by participating in cyclin-D1 and inducing $I L-1 \beta$ production (Hai et al., 2016). In HCC, the molecular platform components of the NLRP3 inflammasome are lost or significantly reduced compared with normal liver (Wei et al., 2014). We found that NLRP3 was upregulated in the LUSC tissues and negatively correlated with survival time. NLRP3 inflammasome could mediate pyroptosis through the cleavage of the GSDM family proteins, and GSDMD is one of main substrate of NLRP3 inflammasome-induced pyroptosis (Martinon et al., 2004). The outcomes revealed the possibility of NLRP3 inflammasome-induced pyroptosis in causing the development of some tumors.

GPX4, a member of the GPX family, is an important peroxide inhibitor protein. More and more explorations showed that GPX4 is involved in the tumorigenesis. Reports have shown that GPX4 expression is obviously higher in the liver biopsy tissue among patients suffering liver cancer than in nontumor tissue (Guerriero et al., 2015). Zhao et al. (Zhao et al., 2017) proposed that GPX4 protein levels are high in glioma tissues and cell lines, and GPX 4 has close relation with the proliferation, migration, and apoptosis of glioma cells. Explorations have shown that conditional GPX4 
knockout in monocytes promotes CASP11 activation and GSDMD-mediated pyroptosis (Corrales et al., 2016).

In tumors, $I L-1 B$ is produced and secreted by a variety of cell types, like immune cells, fibroblasts, or carcinoma cells. As a proinflammatory cytokine that is expressed in primary tumors, $I L-1 B$ has been identified as a potential biomarker in patients with breast carcinoma (Martínez-Reza et al., 2019), $I L-1 B$ is highly expressed in the blood of patients with NSCLC (Kim et al., 2013), which was in consistency with the outcomes. High $I L-1 B$ levels had association with less overall and survival rates in LUSC patients. In conclusion, these nine genes have been confirmed to be related genes involved in tumorigenesis and development in the prognostic model. However, the manner by which these genes interact with each other in the process of pyroptosis remains to be further studied.

To date, full studies have not been performed on pyroptosis, hough we had known some resemblances in apoptosis and some crossover in the mechanism. With the advancement of tumors develop, coexistence and interaction might be seen in various patterns of cellular deaths (Fritsch et al., 2019). For example, in our model, three genes (i.e., CASP4, CASP5, and CASP9) were also identified as key regulators of the apoptotic pathway. Then, we analyzed the DEGs in the various risk groups and revealed that the DEGs got main involvement in leukocyte migration, regulation of immune effector process, and cytokine-cytokine receptor interaction, indicating that dead cells induced an intense inflammation response. Upon the basis of the GO and KEGG analysis, pyroptosis may be inferred to regulate the constitution of tumor immunization micro environment.

\subsection{Correlation Between Pyroptosis and Tumor Immune Microenvironment}

According to our results, except for nature killer (NK) cells, the infiltratory level of immune cells in the high-risk subgroup was normally lower contrast with the low-risk subgroup, suggesting an overall impairment of immunity functionalities in the highrisk group. Our study found the higher proportion of Treg cells in the low-risk group versus in the high-risk group. Treg cells are highly immunosuppressive, and in malignant tumors, these cells promote tumor progression by inhibiting effective antitumor immunity. High Treg infiltration has been observed in tumor tissues (Wolf et al., 2005), and the increase in the number of Treg and the decrease in the ratio of Treg have been associated with poor tumor prognosis (Toker et al., 2018). One likely cause for such difference may be that Treg cells are necessary in regulating the overactive inflammation response resulting from pyroptosis in the tumor microenvironment. In addition, all those 13 immune-associated pathways presented lower activity in the high-risk group versus the low-risk group. Upon the basis of such findings, the low surviving outcomes in high-risk LUSC patients may result from reduced levels of anti-tumor immunity.

At present, few studies have been conducted on pyroptosis in LUSC, especially its mechanism. preliminary exploration was made on the prognosis of such pyroptosis-related genes, providing theory foundation for upcoming explorations. Nevertheless, because of the deficiency in data, we can't determine whether these genes also play a corresponding role in the pyroptosis pathway of LUSC, and this phenomenon deserves further investigation.

\section{CONCLUSION}

In summary, pyroptosis in the LUSC tissues was closely related to LUSC, as most pyroptosis-related genes are expressed differently amid healthy tissues and LUSC tissues. In addition, the score generated based on the risk markers for the nine genes associated with pyroptosis served as an independent risk factor in prediction of OS. The difference between the two risk groups had association with tumor immunity. This exploration provides a novel genetic marker for prediction of prognosis in LUSC and provides an essential foundation for further study of the relation amid genes associated with pyroptosis and immunity in LUSC.

\section{DATA AVAILABILITY STATEMENT}

The datasets presented in this study can be found in online repositories. The names of the repository/repositories and accession number(s) can be found in the article/ Supplementary Material.

\section{AUTHOR CONTRIBUTIONS}

TL performed statistical analysis, and was responsible for the quality control of data and algorithms. HL performed literature research and data interpretation. CD helped data discussion. JL contributed to the study concept and study design. All authors contributed to writing of the manuscript and approved the final version.

\section{ACKNOWLEDGMENTS}

We thank all colleagues involved in the study for their contributions. We acknowledge TCGA database for providing their platforms and contributors for uploading their meaningful datasets.

\section{SUPPLEMENTARY MATERIAL}

The Supplementary Material for this article can be found online at: https://www.frontiersin.org/articles/10.3389/fphar.2022.806995/ full\#supplementary-material 


\section{REFERENCES}

Abe, J., and Morrell, C. (2016). Pyroptosis as a Regulated Form of Necrosis: PI+/ Annexin V-/High Caspase 1/Low Caspase 9 Activity in Cells = Pyroptosis. Circ. Res. 118 (10), 1457-1460. doi:10.1161/CIRCRESAHA.116.308699

Baker, P. J., Boucher, D., Bierschenk, D., Tebartz, C., Whitney, P. G., D’Silva, D. B., et al. (2015). NLRP3 Inflammasome Activation Downstream of Cytoplasmic LPS Recognition by Both Caspase-4 and Caspase-5. Eur. J. Immunol. 45 (10), 2918-2926. doi:10.1002/eji.201545655

Corrales, L., McWhirter, S. M., Dubensky, T. W., Jr, and Gajewski, T. F. (2016). The Host STING Pathway at the Interface of Cancer and Immunity. J. Clin. Invest. 126 (7), 2404-2411. doi:10.1172/JCI86892

Correa, R. G., Milutinovic, S., and Reed, J. C. (2012). Roles of NOD1 (NLRC1) and NOD2 (NLRC2) in Innate Immunity and Inflammatory Diseases. Biosci. Rep. 32 (6), 597-608. doi:10.1042/BSR20120055

Cotterchio, M., Lowcock, E., Bider-Canfield, Z., Lemire, M., Greenwood, C., Gallinger, S., et al. (2015). Association between Variants in Atopy-Related Immunologic Candidate Genes and Pancreatic Cancer Risk. PloS one 10 (5), e0125273. doi:10.1371/journal.pone.0125273

Drilon, A., Rekhtman, N., Ladanyi, M., and Paik, P. (2012). Squamous-cell Carcinomas of the Lung: Emerging Biology, Controversies, and the Promise of Targeted Therapy. Lancet Oncol. 13 (10), e418-26. doi:10.1016/S14702045(12)70291-7

Ferlay, J., Ervik, M., Lam, F., Colombet, M., Mery, L., Piñeros, M., et al. (2020). Global Cancer Observatory: Cancer Today. Lyon: International Agency for Research on Cancer.

Fritsch, M., Günther, S. D., Schwarzer, R., Albert, M. C., Schorn, F., Werthenbach, J. P., et al. (2019). Caspase-8 Is the Molecular Switch for Apoptosis, Necroptosis and Pyroptosis. Nature 575 (7784), 683-687. doi:10.1038/s41586-019-1770-6

Fu, Z., Akula, S., Thorpe, M., and Hellman, L. (2020). Potent and Broad but Not Unselective Cleavage of Cytokines and Chemokines by Human Neutrophil Elastase and Proteinase 3. Int. J. Mol. Sci. 21 (2), 651. doi:10.3390/ijms21020651

Galluzzi, L., López-Soto, A., Kumar, S., and Kroemer, G. (2016). Caspases Connect Cell-Death Signaling to Organismal Homeostasis. Immunity 44 (2), 221-231. doi:10.1016/j.immuni.2016.01.020

Galluzzi, L., Vitale, I., Aaronson, S. A., Abrams, J. M., Adam, D., Agostinis, P., et al. (2018). Molecular Mechanisms of Cell Death: Recommendations of the Nomenclature Committee on Cell Death 2018. Cell Death Differ 25 (3), 486-541. doi:10.1038/s41418-017-0012-4

Gao, J., Qiu, X., Xi, G., Liu, H., Zhang, F., Lv, T., et al. (2018). Downregulation of GSDMD Attenuates Tumor Proliferation via the Intrinsic Mitochondrial Apoptotic Pathway and Inhibition of EGFR/Akt Signaling and Predicts a Good Prognosis in Nonsmall Cell Lung Cancer. Oncol. Rep. 40 (4), 1971-1984. doi:10.3892/or.2018.6634

Guerriero, E., Capone, F., Accardo, M., Sorice, A., Costantini, M., Colonna, G., et al. (2015). GPX4 and GPX7 Over-expression in Human Hepatocellular Carcinoma Tissues. Eur. J. Histochem. 59 (4), 2540. doi:10.4081/ejh.2015.2540

Hai, P., Feng Bo, T., Li, L., Nan Hui, Y., and Hong, Z. (2016). IL-1 $\beta / N F-k b$ Signaling Promotes Colorectal Cancer Cell Growth through miR-181a/PTEN axis. Arch. Biochem. Biophys. 604, 20-26. doi:10.1016/j.abb.2016.06.001

He, W. T., Wan, H., Hu, L., Chen, P., Wang, X., Huang, Z., et al. (2015). Gasdermin D Is an Executor of Pyroptosis and Required for Interleukin-1 $\beta$ Secretion. Cell Res 25 (12), 1285-1298. doi:10.1038/cr.2015.139

Hergueta-Redondo, M., Sarrió, D., Molina-Crespo, Á., Megias, D., Mota, A., RojoSebastian, A., et al. (2014). Gasdermin-B Promotes Invasion and Metastasis in Breast Cancer Cells. PloS one 9 (3), e90099. doi:10.1371/journal.pone.0090099

Hirsch, F. R., Scagliotti, G. V., Mulshine, J. L., Kwon, R., Curran, W. J., Jr, and Wu, L. (2017). Lung Cancer: Current Therapies and New Targeted Treatments. Lancet 389 (10066), 299-311. doi:10.1016/S0140-6736(16)30958-8

Hu, B., Elinav, E., Huber, S., Booth, C. J., Strowig, T., Jin, C., et al. (2010). Inflammation-induced Tumorigenesis in the colon Is Regulated by Caspase-1 and NLRC4. Proc. Natl. Acad. Sci. U S A. 107 (50), 21635-21640. doi:10.1073/ pnas. 1016814108

Kambara, H., Liu, F., Zhang, X., Liu, P., Bajrami, B., Teng, Y., et al. (2018). Gasdermin D Exerts Anti-inflammatory Effects by Promoting Neutrophil Death. Cell Rep 22 (11), 2924-2936. doi:10.1016/j.celrep.2018.02.067
Karki, R., and Kanneganti, T. D. (2019). Diverging Inflammasome Signals in Tumorigenesis and Potential Targeting. Nat. Rev. Cancer 19 (4), 197-214. doi:10.1038/s41568-019-0123-y

Kim, B., Srivastava, S. K., and Kim, S. H. (2015). Caspase- 9 as a Therapeutic Target for Treating Cancer. Expert Opin. Ther. Targets 19 (1), 113-127. doi:10.1517/ 14728222.2014.961425

Kim, J. W., Koh, Y., Kim, D. W., Ahn, Y. O., Kim, T. M., Han, S. W., et al. (2013). Clinical Implications of VEGF, TGF-B1, and IL-1 $\beta$ in Patients with Advanced Non-small Cell Lung Cancer. Cancer Res. Treat. 45 (4), 325-333. doi:10.4143/ crt.2013.45.4.325

Layunta, E., Latorre, E., Forcén, R., Grasa, L., Plaza, M. A., Arias, M., et al. (2018). NOD1 Downregulates Intestinal Serotonin Transporter and Interacts with Other Pattern Recognition Receptors. J. Cel Physiol 233 (5), 4183-4193. doi:10.1002/jcp.26229

Man, S. M., and Kanneganti, T. D. (2015). Regulation of Inflammasome Activation. Immunol. Rev. 265 (1), 6-21. doi:10.1111/imr.12296

Martínez-Reza, I., Díaz, L., Barrera, D., Segovia-Mendoza, M., Pedraza-Sánchez, S Soca-Chafre, G., et al. (2019). Calcitriol Inhibits the Proliferation of TripleNegative Breast Cancer Cells through a Mechanism Involving the Proinflammatory Cytokines IL-1ßand TNF-a. J. Immunol. Res. 2019, 1-11. doi:10.1155/2019/6384278

Martinon, F., Agostini, L., Meylan, E., and Tschopp, J. (2004). Identification of Bacterial Muramyl Dipeptide as Activator of the NALP3/cryopyrin Inflammasome. Curr. Biol. 14 (21), 1929-1934. doi:10.1016/j.cub.2004. 10.027

Mattiuzzi, C., and Lippi, G. (2020). Cancer Statistics: a Comparison between World Health Organization (WHO) and Global Burden of Disease (GBD). Eur. J. Public Health 30 (5), 1026-1027. doi:10.1093/eurpub/ckz216

Nagarajan, K., Soundarapandian, K., Thorne, R. F., Li, D., and Li, D. (2019). Activation of Pyroptotic Cell Death Pathways in Cancer: An Alternative Therapeutic Approach. Transl Oncol. 12 (7), 925-931. doi:10.1016/j.tranon. 2019.04.010

Ozbayer, C., Kurt, H., Bayramoglu, A., Gunes, H. V., Metintas, M., Degirmenci, İ., et al. (2015). The Role of NOD1/CARD4 and NOD2/CARD15 Genetic Variations in Lung Cancer Risk. Inflamm. Res. 64 (10), 775-779. doi:10. 1007/s00011-015-0859-0

Pizato, N., Luzete, B. C., Kiffer, L. F. M. V., Corrêa, L. H., de Oliveira Santos, I., Assumpção, J. A. F., et al. (2018). Omega-3 Docosahexaenoic Acid Induces Pyroptosis Cell Death in Triple-Negative Breast Cancer Cells. Sci. Rep. 8 (1), 1952. doi:10.1038/s41598-018-20422-0

Qiao, L., Wu, X., Zhang, J., Liu, L., Sui, X., Zhang, R., et al. (2019). a-NETA Induces Pyroptosis of Epithelial Ovarian Cancer Cells through the GSDMD/caspase-4 Pathway. FASEB J. 33 (11), 12760-12767. doi:10.1096/fj.201900483RR

Qiu, S., Liu, J., and Xing, F. (2017). 'Hints' in the Killer Protein Gasdermin D: Unveiling the Secrets of Gasdermins Driving Cell Death. Cel Death Differ 24 (4), 588-596. doi:10.1038/cdd.2017.24

Saeki, N., Usui, T., Aoyagi, K., Kim, D. H., Sato, M., Mabuchi, T., et al. (2009). Distinctive Expression and Function of Four GSDM Family Genes (GSDMAD) in normal and Malignant Upper Gastrointestinal Epithelium. Genes Chromosomes Cancer 48 (3), 261-271. doi:10.1002/gcc.20636

Scagliotti, G. V., Parikh, P., von Pawel, J., Biesma, B., Vansteenkiste, J., Manegold, C., et al. (2008). Phase III Study Comparing Cisplatin Plus Gemcitabine with Cisplatin Plus Pemetrexed in Chemotherapy-Naive Patients with AdvancedStage Non-small-cell Lung Cancer. J. Clin. Oncol. 26 (21), 3543-3551. doi:10. 1200/JCO.2007.15.0375

Schiller, J. H., Harrington, D., Belani, C. P., Langer, C., Sandler, A., Krook, J., et al. (2002). Comparison of Four Chemotherapy Regimens for Advanced Nonsmall-cell Lung Cancer. N. Engl. J. Med. 346 (2), 92-98. doi:10.1056/ NEJMoa011954

Shi, J., Gao, W., and Shao, F. (2017). Pyroptosis: Gasdermin-Mediated Programmed Necrotic Cell Death. Trends Biochem. Sci. 42 (4), 245-254. doi:10.1016/j.tibs.2016.10.004

Shi, J., Zhao, Y., Wang, K., Shi, X., Wang, Y., Huang, H., et al. (2015). Cleavage of GSDMD by Inflammatory Caspases Determines Pyroptotic Cell Death. Nature 526 (7575), 660-665. doi:10.1038/nature15514

Shi, J., Zhao, Y., Wang, Y., Gao, W., Ding, J., Li, P., et al. (2014). Inflammatory Caspases Are Innate Immune Receptors for Intracellular LPS. Nature 514 (7521), 187-192. doi:10.1038/nature13683 
Siegel, R. L., Miller, K. D., and Jemal, A. (2018). Cancer Statistics, 2018. CA Cancer J. Clin. 68 (1), 7-30. doi:10.3322/caac.21442

Suarez, G., Romero-Gallo, J., Piazuelo, M. B., Wang, G., Maier, R. J., Forsberg, L. S., et al. (2015). Modification of Helicobacter pylori Peptidoglycan Enhances NOD1 Activation and Promotes Cancer of the Stomach. Cancer Res. 75 (8), 1749-1759. doi:10.1158/0008-5472.CAN-14-2291

Terlizzi, M., Colarusso, C., De Rosa, I., De Rosa, N., Somma, P., Curcio, C., et al. (2018). Circulating and Tumor-Associated Caspase-4: a Novel Diagnostic and Prognostic Biomarker for Non-small Cell Lung Cancer. Oncotarget 9 (27), 19356-19367. doi:10.18632/oncotarget.25049

Toker, A., Nguyen, L. T., Stone, S. C., Yang, S. Y. C., Katz, S. R., Shaw, P. A., et al. (2018). Regulatory T Cells in Ovarian Cancer Are Characterized by a Highly Activated Phenotype Distinct from that in Melanoma. Clin. Cancer Res. 24 (22), 5685-5696. doi:10.1158/1078-0432.CCR-18-0554

Wang, B., and Yin, Q. (2017). AIM2 Inflammasome Activation and Regulation: A Structural Perspective. J. Struct. Biol. 200 (3), 279-282. doi:10.1016/j.jsb.2017. 08.001

Wang, C. J., Tang, L., Shen, D. W., Wang, C., Yuan, Q. Y., Gao, W., et al. (2013). The Expression and Regulation of DFNA5 in Human Hepatocellular Carcinoma DFNA5 in Hepatocellular Carcinoma. Mol. Biol. Rep. 40 (12), 6525-6531. doi:10.1007/s11033-013-2581-8

Wei, Q., Mu, K., Li, T., Zhang, Y., Yang, Z., Jia, X., et al. (2014). Deregulation of the NLRP3 Inflammasome in Hepatic Parenchymal Cells during Liver Cancer Progression. Lab. Invest. 94 (1), 52-62. doi:10.1038/labinvest. 2013.126

Wolf, D., Wolf, A. M., Rumpold, H., Fiegl, H., Zeimet, A. G., Muller-Holzner, E., et al. (2005). The Expression of the Regulatory T Cell-specific Forkhead Box Transcription Factor FoxP3 Is Associated with Poor Prognosis in Ovarian Cancer. Clin. Cancer Res. 11 (23), 8326-8331. doi:10.1158/1078-0432.CCR-051244

Xi, G., Gao, J., Wan, B., Zhan, P., Xu, W., Lv, T., et al. (2019). GSDMD Is Required for Effector CD8+ $\mathrm{T}$ Cell Responses to Lung Cancer Cells. Int. Immunopharmacol 74, 105713. doi:10.1016/j.intimp.2019.105713
Xia, X., Wang, X., Cheng, Z., Qin, W., Lei, L., Jiang, J., et al. (2019). The Role of Pyroptosis in Cancer: Pro-cancer or Pro-"host". Cell Death Dis 10 (9), 650. doi:10.1038/s41419-019-1883-8

Ye, Y., Dai, Q., and Qi, H. (2021). A Novel Defined Pyroptosis-Related Gene Signature for Predicting the Prognosis of Ovarian Cancer. Cell Death Discov 7 (1), 71. doi:10.1038/s41420-021-00451-x

Zhang, T., Li, Y., Zhu, R., Song, P., Wei, Y., Liang, T., et al. (2019). Transcription Factor P53 Suppresses Tumor Growth by Prompting Pyroptosis in Non-smallcell Lung Cancer. Oxid Med. Cel Longev 2019, 8746895. doi:10.1155/2019/ 8746895

Zhao, H., Ji, B., Chen, J., Huang, Q., and Lu, X. (2017). Gpx 4 Is Involved in the Proliferation, Migration and Apoptosis of Glioma Cells. Pathol. Res. Pract. 213 (6), 626-633. doi:10.1016/j.prp.2017.04.025

Zhao, Y., Shi, J., and Shao, F. (2018). Inflammatory Caspases: Activation and Cleavage of Gasdermin-D In Vitro and during Pyroptosis. Methods Mol. Biol. 1714, 131-148. doi:10.1007/978-1-4939-7519-8_9

Conflict of Interest: The authors declare that the research was conducted in the absence of any commercial or financial relationships that could be construed as a potential conflict of interest.

Publisher's Note: All claims expressed in this article are solely those of the authors and do not necessarily represent those of their affiliated organizations, or those of the publisher, the editors and the reviewers. Any product that may be evaluated in this article, or claim that may be made by its manufacturer, is not guaranteed or endorsed by the publisher.

Copyright $\odot 2022 \mathrm{Li}$, Liu, Dong and Lyu. This is an open-access article distributed under the terms of the Creative Commons Attribution License (CC BY). The use, distribution or reproduction in other forums is permitted, provided the original author(s) and the copyright owner(s) are credited and that the original publication in this journal is cited, in accordance with accepted academic practice. No use, distribution or reproduction is permitted which does not comply with these terms. 